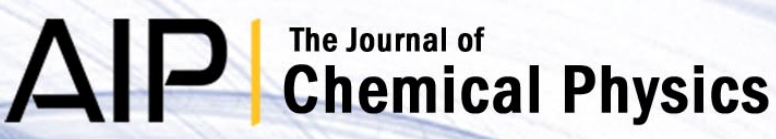

Spectral line shapes in dissipative systems: Molecules adsorbed on metal surfaces

S. Miret-Artés, D. A. Micha, and D. Beksic

Citation: J. Chem. Phys. 114, 4690 (2001); doi: 10.1063/1.1340672

View online: http://dx.doi.org/10.1063/1.1340672

View Table of Contents: http://jcp.aip.org/resource/1/JCPSA6/v114/i10

Published by the American Institute of Physics.

\section{Additional information on J. Chem. Phys.}

Journal Homepage: http://jcp.aip.org/

Journal Information: http://jcp.aip.org/about/about_the_journal

Top downloads: http://jcp.aip.org/features/most_downloaded

Information for Authors: http://jcp.aip.org/authors

\section{ADVERTISEMENT}

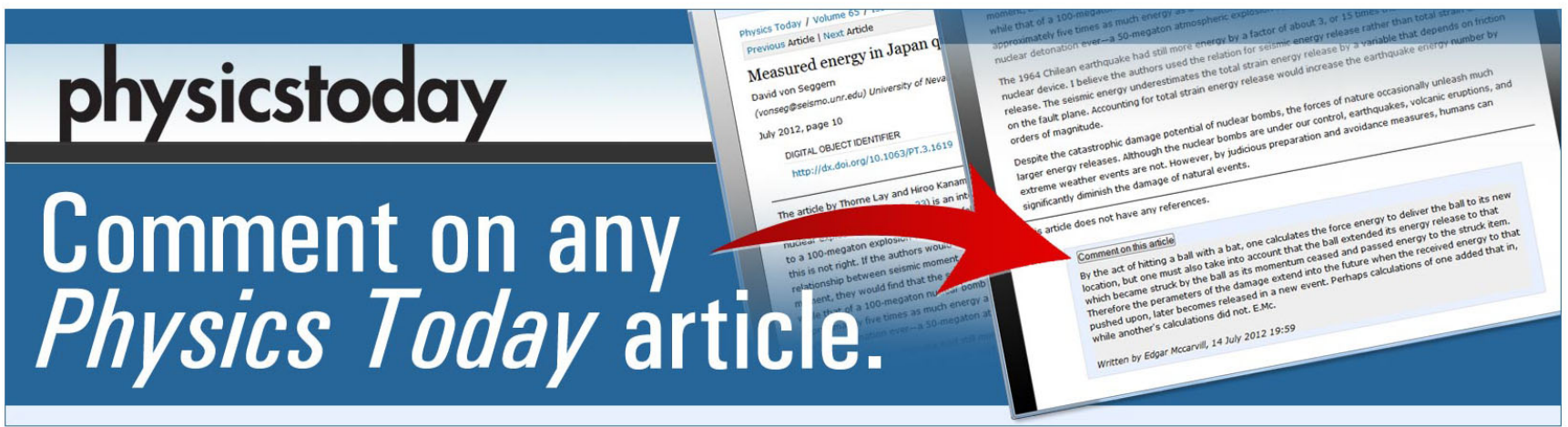




\title{
Spectral line shapes in dissipative systems: Molecules adsorbed on metal surfaces
}

\author{
S. Miret-Artés \\ Instituto de Matemáticas y Física Fundamental, C. S. I. C., Serrano 123, 28006 Madrid, Spain \\ D. A. Micha ${ }^{\text {a) }}$ and D. Beksic \\ Quantum Theory Project, Departments of Chemistry and of Physics, University of Florida, Gainesville, \\ Florida 32611-8435
}

(Received 11 February 2000; accepted 21 November 2000)

\begin{abstract}
The temporal evolution of rates and probabilities of transitions, induced by light between states of a molecule interacting with a dissipative medium, are derived for a Hamiltonian containing an anti-Hermitian term which describes dissipation. Our theoretical treatment introduces a biorthonormal basis set of decaying eigenstates to obtain the rates and probabilities from perturbation theory. The formalism is developed for excitation by continuous and pulsed light. As an example, it is applied to the excitation of molecules adsorbed on solid surfaces, taking into account the dissipation of energy into the solid as the molecule relaxes or is desorbed. (C) 2001 American Institute of Physics. [DOI: 10.1063/1.1340672]
\end{abstract}

\section{INTRODUCTION}

State-to-state rates of transition and the line shapes of spectra are affected by the lifetimes of the involved states. In the case of a molecular system in contact with a bath, or at a solid surface, its excited states decay over time as energy is dissipated into the surroundings. This decay is frequently represented in wave function ${ }^{1}$ and density matrix treatments ${ }^{2}$ by means of phenomenological lifetimes.

An alternative approach to the study of dissipative phenomena starts with the Hamiltonian of the whole system (molecule plus bath) and obtains an effective Hamiltonian for the molecule. The effective Hamiltonian contains new operator terms that describe the dissipation and are functions of the system's degrees of freedom. This approach is therefore more fundamental, and allows access to the lifetimes from molecular properties and interactions. The dissipation is described by an anti-Hermitian term in the Hamiltonian, which in turn leads to complex energy eigenvalues and to decaying states in a time-dependent description. This can be done in a time-dependent framework, starting with the Liouville-von Neumann equation for the density operator to derive generalized Langevin equations, ${ }^{2,3}$ or it can follow from a study of discrete states embedded in continua, as it happens in the coupling of molecules with the electromagnetic field ${ }^{4,5}$ and in collisions of species with internal structure where one introduces optical potentials. ${ }^{6-9}$

Given the non-Hermitian Hamiltonian, the next task is to derive the rates for state-to-state transitions, and related line shapes of spectra. In what follows we develop a straightforward formalism for the temporal dependence of transition rates, consider it for cases where the molecular system is excited by light (both continuous and pulsed), and comment on the application of the formulas to desorption with dissi-

a) Author to whom correspondence should be addressed. pation. Our treatment relates to studies of the optical Bloch equations, where two-state systems are excited by continuous or pulsed light. ${ }^{10-13}$ In our case we deal with a multilevel system and include dissipative effects, within a perturbative treatment. This is not a trivial exercise in perturbation theory, because the states are nonstationary and we need the longtime behavior of the system.

In this paper we consider physical situations where a molecule adsorbed on a metal surface is excited by light and makes a transition into a different vibrational-rotational or electronic state, while energy is dissipated into the solid surface as phonons or electron-hole pairs. ${ }^{14-18}$ We have recently treated this situation with a model which incorporates the coupling of a primary region made up of the adsorbate and binding atoms in the substrate, with a secondary region containing the remaining substrate, in a density matrix approach using a time-dependent self-consistent field factorization and a stochastic description of the secondary region. ${ }^{19-21}$ The functional form of the dissipation was given in terms of the time-correlation of atomic displacements in the secondary region, related to phonons, and of electronic charge density fluctuations, related to electron-hole excitations. ${ }^{19}$ In one of the examples treated later on, for electronic excitation by a femtosecond pulse of UV light, the substrate is a metal with fast electronic relaxation but slow vibrations, which allows us to neglect the dissipation from phonon excitation. Other earlier treatments of surface phenomena such as desorption have also included an optical potential in the Hamiltonian. $^{22}$

\section{TRANSITION PROBABILITIES AND RATES}

The purpose of this section is to present a detailed derivation for the transition probabilities and rates for a dissipative system interacting with an external radiation field in a continuum or pulsed regime. Splitting the total system into two subsystems (primary, $p$, and secondary, $s$, regions), the 
total Hamiltonian can be written as $H_{T}=H_{p}+H_{s}+H_{p s}$, where $H_{p s}$ represents the interaction between both regions. Each region drives the other one, and fluctuation and dissipation effects in the $p$-region can be described with a primary effective Hamiltonian. This leads to time-dependent and complex-valued effective potentials that describe the broadening of energy levels due to the coupling with the bath or secondary region. For fast fluctuation and dissipation, the primary effective Hamiltonian $F_{p}$ simplifies to the form ${ }^{19,22}$

$$
F_{p}=F_{p}^{0}+H_{p L}=H_{p}-\frac{i}{2} W_{p}+H_{p L},
$$

where $W_{p}$ is a positive Hermitian operator, due in our case to the energy dissipation into the metal. The interaction $H_{p L}$ of the radiation field with the molecule (which is part of the primary region), can be written in the dipole approximation as

$$
H_{p L}(t)=-\mathbf{D}_{p} \cdot \mathcal{E}(t)=-D_{p} \mathcal{E}(t),
$$

where $\mathbf{D}_{p}$ is the effective electric dipole vector in the primary region and $D_{p}$ is its projection on the polarization direction of $\mathcal{E}(t)$, the electric field of the light, which is assumed turned on at time $t=0$. For adsorbates, this coupling may occur directly between the light and the bond being excited, or indirectly with excitation of the substrate by light absorption followed by transfer of the excitation to the bond. When excitation is direct, the dipole operator in Eq. (2) depends only on the coordinates of the $p$-region, $D_{p}^{0}$; when the excitation is indirect, this becomes an effective dipole operator $\Delta_{p}$ which also depends on the dynamics of the $s$-region excitation, and may vary with time, as found in a recent stochastic treatment of photodesorption. ${ }^{20,21}$ The expressions that follow are derived for a time-independent dipole operator, but are easily generalized to time-dependent ones.

A basis set of stationary states of the primary region may be introduced to describe this region, in the absence of coupling with the secondary region and the light. They satisfy the time-independent equation $H_{p}\left|\phi_{n}^{0}\right\rangle=E_{n}^{0}\left|\phi_{n}^{0}\right\rangle$, and form an orthonormal set. We construct from them the nonstationary states of the primary region coupled to the secondary one. In what follows we drop the subindex $p$, write $F_{p}^{0}$ $=F_{0}, H_{p}=H, W_{p}=W$, and $H_{p L}=V_{L}$, keeping in mind that the light interacts only with the dipole, $D_{p}=D$ which would equal the primary dipole $D_{p}^{0}$ for direct excitation, or an effective dipole operator $\Delta_{p}$ for indirect excitation.

The zero-order Hamiltonian $F_{0}$ is not Hermitian and therefore it has complex energy eigenvalues. It is well known that for this kind of operators, the orthogonality properties and completeness of its eigenstates $\left|\psi_{n}^{0}\right\rangle$ are different from those derived from hermitian Hamiltonians. ${ }^{23}$ Thus we have that

$$
F_{0}\left|\psi_{n}^{0}\right\rangle=\left(E_{n}-i \Gamma_{n} / 2\right)\left|\psi_{n}^{0}\right\rangle
$$

which we can be formally solved for the states, introducing the notation $E_{n}=E_{n}^{0}+\Delta_{n}-i \Gamma_{n} / 2$, to obtain

$$
\left|\psi_{n}^{0}\right\rangle=\left|\phi_{n}^{0}\right\rangle+\left(E_{n}^{0}-H+i \epsilon\right)^{-1}\left[-i\left(W-\Gamma_{n}\right) / 2-\Delta_{n}\right]\left|\psi_{n}^{0}\right\rangle
$$

with $\epsilon \rightarrow 0+$. Here $\Delta_{n}$ and $\Gamma_{n}$ are of first order in the magnitude $\|W\|$. Similarly we have that $F_{0}^{\dagger}\left|\bar{\psi}_{n}^{0}\right\rangle=\left(E_{n}\right.$ $\left.+i \Gamma_{n} / 2\right)\left|\bar{\psi}_{n}^{0}\right\rangle$. The eigenstates $\left\{\bar{\psi}_{n}^{0}, \psi_{m}^{0}\right\}$ form a biorthogonal set, that is, $\left\langle\bar{\psi}_{n}^{0} \mid \psi_{m}^{0}\right\rangle=\delta_{n m}$, and the resolution of the identity is

$$
\hat{I}=\sum_{n}\left|\psi_{n}^{0}\right\rangle\left\langle\bar{\psi}_{n}^{0}\right|
$$

The eigenstates of $F_{0}$ are not orthogonal and their overlaps are given by

$$
\left\langle\psi_{n}^{0} \mid \psi_{m}^{0}\right\rangle=S_{n m}=\delta_{n m}+\mathcal{O}_{n m}(\|W\|)
$$

insofar the overlap correction is of order $W$. Therefore the eigenstates $\left|\psi_{n}^{0}\right\rangle$ are approximately orthonormal when $\mathcal{O}(\|W\|)$ is small compared with $\mathcal{O}(\|H\|)$. The resolution of the identity is alternatively written, with a matrix notation for the overlap, as

$$
\hat{I}=\sum_{m n}\left|\psi_{n}^{0}\right\rangle\left(\mathbf{S}^{-1}\right)_{n m}\left\langle\psi_{m}^{0}\right| .
$$

Transition amplitudes can be obtained from the time dependent states

$$
\begin{aligned}
\left|\Psi_{m}^{0}(t)\right\rangle & =\exp \left(-i F_{0} t / \hbar\right)\left|\psi_{m}^{0}\right\rangle \\
& =\exp \left[-i\left(E_{m}-i \Gamma_{m} / 2\right) t / \hbar\right]\left|\psi_{m}^{0}\right\rangle
\end{aligned}
$$

as these states change in time due to their coupling to the light. The probability $\left|\left\langle\psi_{m}^{0}(t) \mid \psi_{m}^{0}(t)\right\rangle\right|^{2}$ shows that $\Gamma_{m}$ gives the decay rate for state $m$, and that $\hbar / \Gamma_{m}$ is its decay time constant.

The electric fields of present interest are weak. We assume that the dissipation terms are also small, but need their effect over long times. Therefore, we treat the dynamics in the primary region within first order perturbation theory in the field, but must keep the higher order terms in the dissipation within the complex phase of the decaying states. Thus starting with the time-dependent Schrödinger equation,

$$
i \hbar \frac{\partial}{\partial t}|\Psi(t)\rangle=F(t)|\Psi(t)\rangle
$$

we integrate it from $t=0$ to find for the primary region wave function, to first order in the coupling with the light and for an initial unperturbed state $\left|\Psi_{m}^{0}(t)\right\rangle$, the expression

$$
\begin{aligned}
\left|\Psi_{m}(t)\right\rangle= & \left|\Psi_{m}^{0}(t)\right\rangle-\frac{i}{\hbar} \int_{0}^{t} \exp \left[-i F_{0}\left(t-t^{\prime}\right) / \hbar\right] \\
& \times V_{L}\left(t^{\prime}\right)\left|\Psi_{m}^{0}\left(t^{\prime}\right)\right\rangle d t^{\prime} .
\end{aligned}
$$

Expectation values of properties can be obtained from this wave function. In particular, the survival probability of the initial state $\left|\Psi_{m}(0)\right\rangle=\left|\psi_{m}^{0}\right\rangle$ follows from $P_{m}^{s}(t)$ $=\left|\left\langle\Psi_{m}^{0}(0) \mid \Psi_{m}(t)\right\rangle\right|^{2}$ and the average interaction energy with the field results from $\left\langle V_{L}(t)\right\rangle_{m}$ $=\left\langle\Psi_{m}(t)\left|V_{L}(t)\right| \Psi_{m}(t)\right\rangle$. This gives the linear response of the system to the applied electric field. ${ }^{24}$ In effect, it can be written as $\left\langle V_{L}(t)\right\rangle_{m}=-\mathcal{E}(t)\langle D(t)\rangle_{m}$ with 


$$
\begin{aligned}
\langle D(t)\rangle_{m}= & \langle D(t)\rangle_{m}^{0}+\int_{-\infty}^{\infty} d t^{\prime} \chi_{m}\left(t^{\prime}, t\right) \mathcal{E}\left(t^{\prime}\right), \\
\langle D(t)\rangle_{m}^{0}= & \left\langle\Psi_{m}^{0}(t)|D| \Psi_{m}^{0}(t)\right\rangle \\
= & \exp \left(-\Gamma_{m} t / \hbar\right) D_{m m}, \\
\chi_{m}\left(t^{\prime}, t\right)= & 2 \hbar^{-1} \theta\left(t-t^{\prime}\right) \exp \left(-\Gamma_{m} t / \hbar\right) \\
& \times \sum_{n, j} D_{m n}\left(\mathbf{S}^{-1}\right)_{n j} D_{j m} \\
& \times \exp \left[-\gamma_{n m}\left(t^{\prime}-t\right)\right] \sin \left[\omega_{n m}\left(t^{\prime}-t\right)\right]
\end{aligned}
$$

in terms of the response function $\chi_{m}\left(t, t^{\prime}\right)$. Its Fourier transform with respect to $t-t^{\prime}$ can be related to absorption line shapes at each time $t$. Here we have used an expansion in the basis of unperturbed decaying states which requires the overlap matrix, $\theta(t)$ is the Heaviside step function, and the matrix elements of the dipole operator are written as $D_{n m}$ $=\left\langle\psi_{n}^{0}|D| \psi_{m}^{0}\right\rangle$, and finally $\hbar \omega_{n m}=E_{n}-E_{m}$, and $\hbar \gamma_{n m}$ $=\left(\Gamma_{m}+\Gamma_{n}\right) / 2$.

It is also possible to define state-to-state transition amplitudes $A_{m \rightarrow n}(t)$ for the transition from the $m$ state to the $n(\neq m)$ state as

$$
\begin{aligned}
A_{m \rightarrow n}(t)= & \left\langle\Psi_{n}^{0}(t) \mid \Psi_{m}(t)\right\rangle \\
= & S_{n m} e^{i\left(\omega_{n m}+i \gamma_{n m}\right) t} \\
& -\frac{i}{\hbar} \int_{0}^{t} d t^{\prime}\left\langle\psi_{n}^{0}\left|V_{L}\left(t^{\prime}\right)\right| \psi_{m}^{0}\right\rangle e^{i\left(\omega_{n m}+i \gamma_{n m}\right) t^{\prime}} \\
= & S_{n m} e^{i\left(\omega_{n m}+i \gamma_{n m}\right) t}-\left[T_{m \rightarrow n}^{(+)}(t)+T_{m \rightarrow n}^{(-)}(t)\right] .
\end{aligned}
$$

This equation has been obtained by using Eq. (10) and is valid to first order in the perturbation. The first term shows that a transition amplitude appears even in the absence of light, due to the finite lifetime of the states. However it is usually negligible compared with the following two terms, corresponding respectively to light absorption and stimulated emission. Time-dependent transition probabilities follow from $P_{m \rightarrow n}(t)=\left|A_{m \rightarrow n}(t)\right|^{2}$.

In analogy with the case of a Hermitian Hamiltonian, it is also possible to define a state to state rate ${ }^{25}$ as

$$
R_{m \rightarrow n}(t)=\frac{d}{d t}\left|A_{m \rightarrow n}(t)\right|^{2} .
$$

Thus, if $E_{m}<E_{n}$ and only the absorption process is important, then $R_{m \rightarrow n}^{\mathrm{abs}}(t) \simeq d\left|T_{m \rightarrow n}^{(+)}(t)\right|^{2} / d t$. The total absorption probability is obtained integrating over all times as in

$$
P_{m \rightarrow n}^{\mathrm{abs}}=\int_{0}^{\infty} d t R_{m \rightarrow n}^{\mathrm{abs}}(t)=\left|T_{m \rightarrow n}^{(+)}(\infty)\right|^{2}-\left|T_{m \rightarrow n}^{(+)}(0)\right|^{2} .
$$

To further develop the theory we have to assume an explicit form for the coupling $V_{L}$. Two cases will be considered: photon excitation by a continuous light source, such as a continuous wave (cw) laser, and excitation by a light pulse (or pulsed laser).

\section{PHOTOEXCITATION BY CONTINUOUS AND PULSED LIGHT}

\section{A. Photoexcitation by continuous light}

We first consider excitation by a weak continuous light field started at $t=0$. In this case the coupling takes the form, in the dipole approximation,

$$
V_{L}(t)=-D \mathcal{E}_{0} \cos \left(\omega_{0} t+\varphi\right),
$$

where we assume that light of frequency $\omega_{0}$, electric field strength $\mathcal{E}_{0}$ and phase $\varphi$ is linearly polarized and $D$ is the dipole operator projected on the polarization direction, and let $\mathcal{E}(t)=0$ for $t<0$. Two perturbation terms appear by substituting this equation into Eq. (12) and carrying out the integration over time; the first one is large for stimulated light emission $\left(\omega_{n m}=-\omega_{0}\right)$ and the second one is large for light absorption $\left(\omega_{n m}=\omega_{0}\right)$,

$$
\begin{aligned}
A_{m \rightarrow n}(t)= & S_{n m} e^{i\left(\omega_{n m}+i \gamma_{n m}\right) t}+\left(D_{n m} \mathcal{E}_{0} / 2 \hbar\right) \\
& \times\left[e^{i \varphi} \frac{e^{i\left(\omega_{n m}+\omega_{0}+i \gamma_{n m}\right) t}-1}{\left(\omega_{n m}+\omega_{0}+i \gamma_{n m}\right)}\right. \\
& \left.+e^{-i \varphi} \frac{e^{i\left(\omega_{n m}-\omega_{0}+i \gamma_{n m}\right) t}-1}{\left(\omega_{n m}-\omega_{0}+i \gamma_{n m}\right)}\right] .
\end{aligned}
$$

The first term is small because it contains the product of an exponentially decreasing function of time and the overlap, which is of the order of $W$. We shall therefore neglect this term when the square modulus transition amplitudes are evaluated to obtain more compact formuli for the observables. The absorption probability is given by

$$
\begin{aligned}
& \left|A_{m \rightarrow n}^{\mathrm{abs}}(t)\right|^{2} \simeq\left|T_{m \rightarrow n}^{(+)}(t)\right|^{2}=\left(\frac{\mathcal{E}_{0}}{2 \hbar}\right)^{2} \\
& \quad \times\left|D_{n m}\right|^{2} \frac{1+e^{-\gamma_{n m} t}\left[e^{-\gamma_{n m} t}-2 \cos \left(\omega_{n m}-\omega_{0}\right) t\right]}{\left(\omega_{n m}-\omega_{0}\right)^{2}+\gamma_{n m}^{2}}
\end{aligned}
$$

and the state-to-state rate can be obtained now from Eq. (13) as

$$
\begin{aligned}
R_{m \rightarrow n}^{\mathrm{abs}}(t)= & 2\left(\mathcal{E}_{0} /(2 \hbar)\right)^{2}\left|D_{n m}\right|^{2} e^{-\gamma_{n m} t} \\
& \times\left\{\left(\omega_{n m}-\omega_{0}\right) \sin \left[\left(\omega_{n m}-\omega_{0}\right) t\right]\right. \\
& \left.+\gamma_{n m} \cos \left[\left(\omega_{n m}-\omega_{0}\right) t\right]-\gamma_{n m} e^{-\gamma_{n m} t}\right\} \\
& \times\left[\left(\omega_{n m}-\omega_{0}\right)^{2}+\gamma_{n m}^{2}\right]^{-1},
\end{aligned}
$$

which generalizes known expressions. In the case of no dissipation, with $\gamma_{n m}=0$, this reduces to the well known result for transitions induced by a harmonic perturbation at time $t ;{ }^{1}$ in the limit of $t \rightarrow \infty$ we further recover the standard result for transition rates, which contain a $\delta$-function factor for energy conservation. $^{26}$

Finally, the total absorption probability can be readily obtained by integration over time according to Eq. (14) and Eq. (18) as

$$
P_{m \rightarrow n}^{\mathrm{abs}}\left(\omega_{0}\right)=\left(\frac{\mathcal{E}_{0}}{2 \hbar}\right)^{2} \frac{\left|D_{n m}\right|^{2}}{\left(\omega_{n m}-\omega_{0}\right)^{2}+\gamma_{n m}^{2}} .
$$


Multiplying this probability by the decay constant $\gamma_{n m}$ we obtain a total transition rate with a Lorentzian shape. This follows from the dissipative lifetime of the states. Lorentzian line shapes arise also from radiative lifetimes, in the theory of photoexcitation, when this is developed to second order or higher in the molecule-light coupling. The contribution of radiative lifetimes could be added in our treatment using higher order perturbation theory in the electric field. ${ }^{1}$

\section{B. Photoexcitation by a light pulse}

For the light pulse, we assume a Gaussian function centered at the time $t_{0}$, with a width $a$, and frequency $\omega_{0}$, chosen so that again we can assume that $V_{L}=0$ for $t<0$. Thus the coupling $V_{L}$ takes now the following form (for polarized light parallel to the dipole moment of the primary region)

$$
V_{L}=-D \mathcal{E}(t)=-D \mathcal{E}_{0} e^{-\left(t-t_{0}\right)^{2} / a^{2}} \cos \left(\omega_{0} t+\varphi\right)
$$

with $\mathcal{E}_{0}$ the amplitude of the electric field. A procedure similar to what follows could be implemented for other pulse shapes.

Introducing Eq. (20) into the transition amplitude Eq. (12), we obtain

$$
\begin{aligned}
A_{m \rightarrow n}(t)= & S_{n m} e^{i\left(\omega_{n m}+i \gamma_{n m}\right) t}+\frac{i}{\hbar} D_{n m} \mathcal{E}_{0} \\
& \times \int_{0}^{t} e^{i\left(\omega_{n m}+i \gamma_{n m}\right) t^{\prime}} e^{-\left(t^{\prime}-t_{0}\right)^{2} / a^{2}} \\
& \times \cos \left(\omega_{0} t^{\prime}+\varphi\right) d t^{\prime} .
\end{aligned}
$$

Decomposing the cos into exponentials, two terms again arise corresponding to absorption and stimulated emission of light, as in Eq. (12). Given a transition frequency and small detuning, only one of these two must be considered, while the other is negligible. Thus for the absorption process we have that (for $t \geqslant 0$ ),

$$
\begin{aligned}
A_{m \rightarrow n}^{\mathrm{abs}}(t)= & S_{n m} e^{i\left(\omega_{n m}+i \gamma_{n m}\right) t}+T_{m \rightarrow n}^{(+)}\left(t ; \omega_{0}\right) \\
T_{m \rightarrow n}^{(+)}\left(t ; \omega_{0}\right) & =C_{n m} \int_{0}^{t} d s e^{i\left(\omega_{n m}-\omega\right) s} e^{-\gamma_{n m^{s}} e^{-\left(s-t_{0}\right)^{2} / a^{2}}} \\
& =Y_{m \rightarrow n}^{(+)}\left(t ; \omega_{0}\right)-Y_{m \rightarrow n}^{(+)}\left(0 ; \omega_{0}\right)
\end{aligned}
$$

with $C_{n m}=i \mathcal{E}_{0} D_{n m} \exp (-i \varphi) /(2 \hbar)$. Analogously, for the emission process we have a similar expression with $T_{m \rightarrow n}^{(-)}(t)$ obtained replacing above $\omega_{0}$ and $\varphi$ by $-\omega_{0}$ and $-\varphi$, respectively, applicable to the case $E_{m}>E_{n}$.

The integral in Eq. (22) can be solved analytically in terms of the error function erf, ${ }^{27}$ and is given by

$$
\begin{aligned}
Y_{m \rightarrow n}^{(+)}\left(t ; \omega_{0}\right)= & -(\sqrt{\pi} a / 2) \operatorname{erf}\left[q\left(t ; \omega_{0}\right)\right] \\
& \times e^{-a^{2}\left(\omega_{n m}-\omega_{0}\right)^{2} / 4+a^{2} \gamma_{n m}^{2} / 4-\gamma_{n m} t_{0}} \\
& \times e^{i\left(\omega_{n m}-\omega_{0}\right)\left(t_{0}-a^{2} \gamma_{n m} / 2\right)}
\end{aligned}
$$

with the definition

$$
q\left(t ; \omega_{0}\right)=\frac{\gamma_{n m} a}{2}+\frac{t-t_{0}}{a}+i \frac{\left(\omega_{0}-\omega_{n m}\right) a}{2} .
$$

Similar expressions are obtained for $Y_{m \rightarrow n}^{(-)}(t)$ with $\left(\omega_{0}, \varphi\right)$ replaced above by $\left(-\omega_{0},-\varphi\right)$.

The time dependent absorption probability on- or offresonance is expressed by $P_{m \rightarrow n}^{\text {abs }}(t)=\left|A_{m \rightarrow n}^{\text {abs }}(t)\right|^{2}$ $=\left|T_{m \rightarrow n}^{(+)}\left(t ; \omega_{0}\right)\right|^{2}$. This provides a simple and compact expression which can be readily applied to Gaussian pulses, and generalizes previous results using stationary states. ${ }^{11,12,13}$ Rates and final probabilities follow from Eqs. (13) and (14), respectively.

\section{MODEL CALCULATIONS FOR ADSORBATE EXCITATIONS}

Here we present results for a model applicable to the vibrational and vibrational-electronic excitation of a molecule adsorbed on a metal surface, such as $\mathrm{CO}$ adsorbed on $\mathrm{Cu}(001)$, following absorption of visible/UV light. This leads to the electronic excitation of the substrate, which then transfers energy to the adsorbate and excites it into higher vibrational levels of the same electronic state, or to higher vibrational-electronic states, as follows from theoretical interpretations ${ }^{14,17,15}$ of experiments. A recently proposed model, ${ }^{19,20}$ which gives results in good agreement with femtosecond experiments on photodesorption yields, makes use of an effective dipole operator in the adsorbate (or $p$-) region, derived from a model based on the evidence ${ }^{18,16}$ that the electronic response and relaxation in the substrate are fast, so that the effective electric field coupling to the adsorbate dipole has the same duration as the original pulse, and a distribution of wavelengths containing the range of resonance absorption frequencies of the adsorbate region. We use in what follows physical parameters and experimental conditions with orders of magnitude suggested by $\mathrm{CO} / \mathrm{Cu}(001)$, as typical values for our model calculations.

The light frequency is chosen to have a phase $\varphi$ $=-\pi / 2$, providing continuity for $\mathcal{E}(t)$ at $t=0$. The strength of the electric field exciting the adsorbate is expressed in terms of the laser fluence $F$ and the pulse half-width $a$ as $\mathcal{E}_{0}=2 F^{1 / 2}\left[1 /\left(2 \pi a^{2}\right)\right]^{1 / 4}$, chosen so that the fluence of the pulse is the same irrespective of its width. The value for the pulse is $F=1.0 \mathrm{~mJ} / \mathrm{cm}^{2}=6.418 \times 10^{-3}$ a.u., and $a$ and $t_{0}$ are chosen to describe the pulse half width for which we take $a=t_{0} / 2=50 \mathrm{fs}=2.067 \times 10^{3}$ a.u.

For vibrational excitation in the ground electronic state $g$ by continuous light, such as excitation of a dipole-allowed mode of $\mathrm{CO}$ on $\mathrm{Cu}(001)$, the lifetimes of vibrational states $m=(g, v)$ and $n=\left(g, v^{\prime}\right)$ come from their decay into electronic excitations of the conduction band electrons and into phonon creation in the substrate. ${ }^{14,15,16}$ The excitation could result from direct absorption of infrared light by a dipoleallowed normal mode or, as considered next, from indirect excitation of vibrations by continuous UV light which is first absorbed by the metal and is then partly transferred as infrared energy of frequency $\omega_{0}$ to the adsorbate bond. We choose as model parameters for the indirect mechanism $\hbar \omega_{n m}=20 \mathrm{meV}=7.358 \times 10^{-4}$ a.u., values of $\hbar / \Gamma_{m}=\hbar / \Gamma_{n}$ $=5.0 \mathrm{ps}=2.067 \times 10^{5}$ a.u. for the lifetimes of the states $\mathrm{m}$ and $n$, from which it follows that $\gamma_{n m}=0.2 \mathrm{ps}^{-1}=4.8379$ $\times 10^{-6}$ a.u. for the transition decay width, and an effective transition dipole $D_{n m}=4.0 \times 10^{-2}$ a.u. 


\section{Scaled rate $\mathrm{R}$}

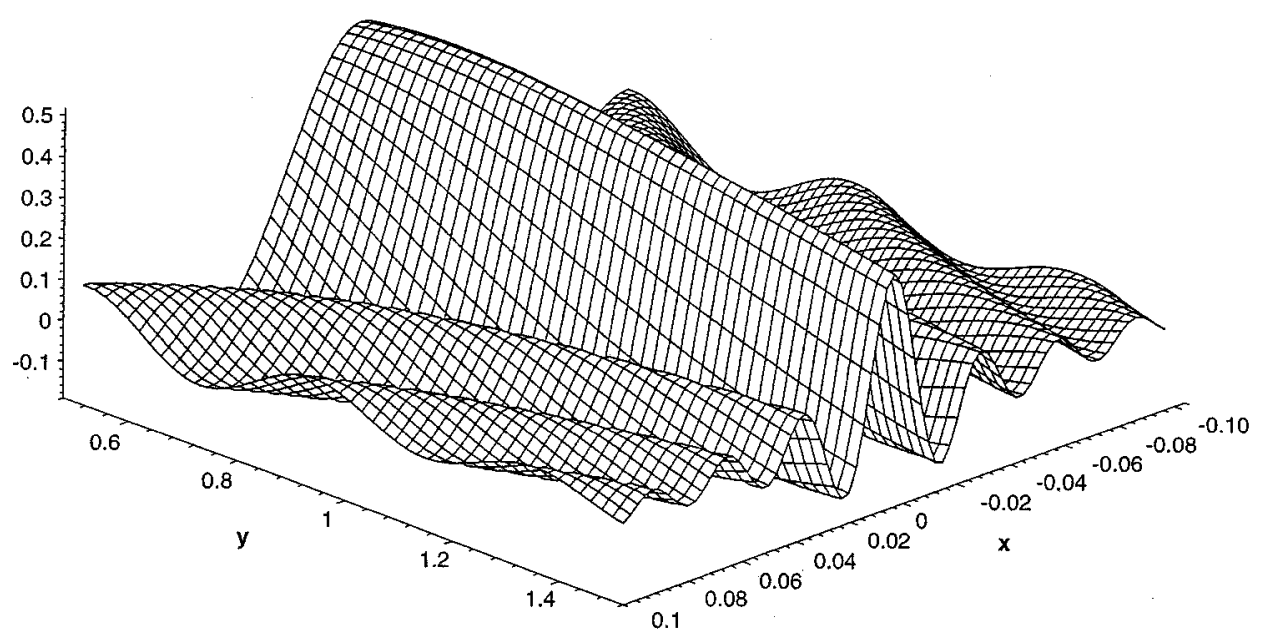

FIG. 1. Scaled rate of transition $R$ for excitation by continuous infrared light, vs the scaled frequency detuning $x$ and scaled time $y$ (see definitions in the text), for the parameters applying to vibrational excitation.

Figure 1 shows the scaled absorption rate $R$ $=R_{m \rightarrow n}^{\mathrm{abs}} \gamma_{n m}(2 \hbar)^{2} /\left[2\left(\mathcal{E}_{0}\left|D_{n m}\right|\right)^{2}\right]$ from Eq. (18). Here we use as variables the detuning $x=\left(\omega_{0}-\omega_{n m}\right) / \omega_{n m}$, and the scaled time $y=\gamma_{n m} t$. We find, at a given time, a large central positive rate peak and oscillatory side bands corresponding to both $m \rightarrow n$ and $n \rightarrow m$ transitions. As the time becomes larger than $\gamma_{n m}^{-1}$, the rate concentrates at a narrower central peak, while its distribution over frequencies spreads into oscillating wings.

For vibrational-electronic excitation of the adsorbate bond by a short UV pulse, the mechanism involves electronic excitations into both the conduction and unoccupied electron energy bands of the substrate; this energy is transferred into the adsorbate region and promotes near resonance $m \rightarrow n$ transitions. The initial state $m=(g, v)$ is a vibrational state $v$ in the ground electronic state $g$ while $n=\left(e, v^{\prime}\right)$ refers to a vibrational state $v^{\prime}$ in an excited electronic state $e$. We use as typical magnitudes $\hbar \omega_{n m}=1.0 \mathrm{eV}=3.675$ $\times 10^{-2}$ a.u., lifetimes $\hbar / \Gamma_{m}=5.0 \mathrm{ps}$ for the ground electronic state and $\hbar / \Gamma_{n}=50.0 \mathrm{fs}$ for the excited electronic state, corresponding to $2.067 \times 10^{5}$ a.u. and $2.067 \times 10^{3}$ a.u., respectively, and a transition dipole $D_{n m}=0.26$ a.u. This gives a transition decay width $\gamma_{n m}=2.4432 \times 10^{-4}$ a.u.

Results have been obtained from Eq. (22) for a pulse of width $a=50 \mathrm{fs}$ and a fluence of $F=1.0 \mathrm{~mJ} / \mathrm{cm}^{2}$, shown in Fig. 2. This displays the scaled absorption probability $P$ $=P_{m \rightarrow n}^{(\text {abs }} /\left(C_{n m}^{2} a^{2}\right)$ vs the same scaled time and detuning we introduced before. At resonance, for zero detuning, the probability is found to increase as time advances. The increase is less pronounced off-resonance, and a small maximum appears as time increases at off-resonance.

\section{CONCLUSION}

We have shown that time-dependent perturbation theory, and the theory of linear response, can be used to describe the temporal evolution of transitions between decaying states, provided one introduces biorthogonal basis sets of a nonHermitian Hamiltonian with dissipative energy terms. Expressions could then be derived for time-dependent transition amplitudes and rates which depend on the decay times. They reduce to well known results when the state decay times become infinite (stationary states) and also when rates are integrated over all times to recover line shapes in the frequency domain.

Two special cases were developed, corresponding to excitation by continuous light and by a light pulse. In the first case we recovered the well-known energy conserving transition rates, and found a Lorentzian line shape for total absorption probabilities due to the presence of dissipation. Expressions for excitation by light pulses lead to new results for the temporal dependence of rates, presented here for both zero and nonzero detuning. When the pulse is gaussian, our results involve the error function of time. These also appear in the solutions of the optical Bloch equations for two state systems $;^{13}$ here we have included the effects of finite decay times, to first order in the perturbing electric field.

We have applied the derived formulas to model calculations of adsorbate excitations. Excited molecular states of adsorbed molecules decay through couplings to the solid surface substrates, and therefore have finite lifetimes which can be described by decay times. We parametrized our models with physical values of orders of magnitude suggested by the 


\section{Scaled Probability P}

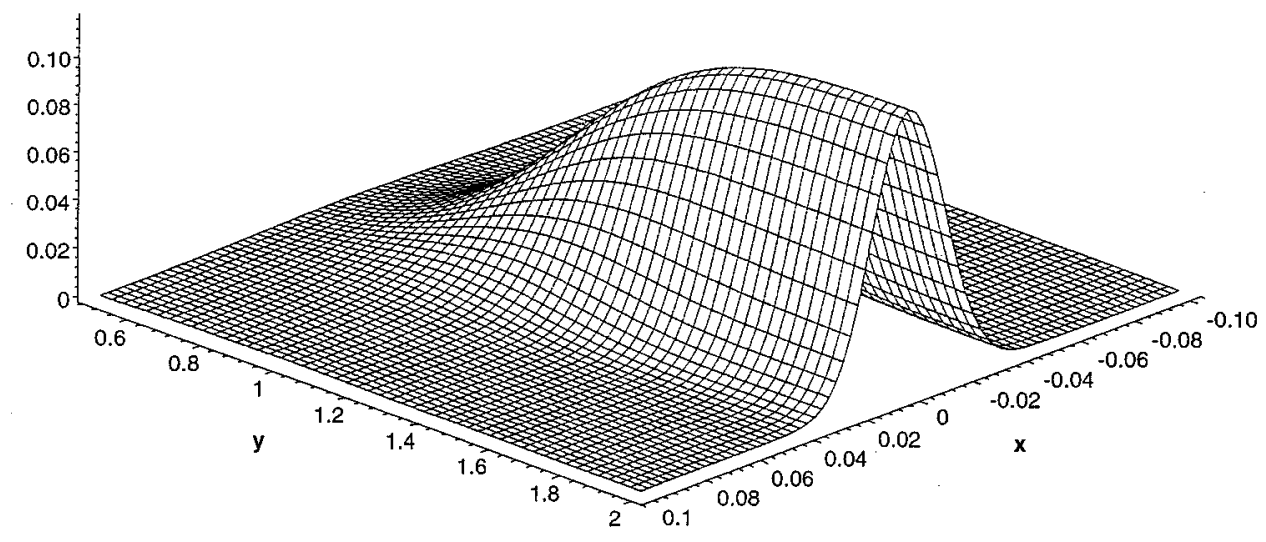

FIG. 2. Scaled probability of transition $P$ for excitation by a femtosecond visible light pulse, vs the scaled frequency detuning $x$ and scaled time $y$ (see definitions in the text), for the parameters applying to electronic-vibrational excitation.

$\mathrm{CO} / \mathrm{Cu}(001)$ system and obtained rates vs time and vs the frequency of the carrying light wave. For vibrational excitation by continuous UV light, we found that the presence of quantal decay times leads to rates which oscillate with both time and light frequency, and decrease exponentially for long times. For excitation by femtosecond pulses, the formulas give simple expressions showing that the absorption probability increases with time and peaks at resonance, as expected. The derivations allow calculations for varying dissipation strengths, embodied in the transition widths $\gamma_{n m}$.

\section{ACKNOWLEDGMENTS}

The work of D.A.M. has been partly supported by the National Science Foundation and Office of Naval Research in the USA and by the Alexander von Humboldt Foundation in Germany. S.M.A. has been partly supported by the DGICYT (Spain) under Contract No. PB95-0071 and gratefully acknowledges the support by the QTP at the University of Florida during his stays. They thank Professors J. P. Toennies and G. Diercksen for their hospitality at the MaxPlanck-Institut für Strömungsforschung (Göttingen), and für Astrophysik (Munich), respectively.

${ }^{1}$ R. Loudon, The Quantum Theory of Light (Clarendon, Oxford, 1973), Chap. 8.

${ }^{2}$ K. Blum, Density Matrix Theory and Applications, 2nd ed. (Plenum, New York, 1996), Chap. 8.

${ }^{3}$ B. J. Berne and R. Pecora, Dynamic Light Scattering (Wiley, New York, 1976), Chap. 11.

${ }^{4}$ C. Cohen-Tannoudji, J. Dupont-Roc, and G. Grynberg, Atom-Photon Interactions (Wiley, New York, 1992), Chap. IV.
${ }^{5}$ S. Mukamel Principles of Nonlinear Optical Spectroscopy (Oxford University Press, Oxford, 1995), Chap. 6.

${ }^{6}$ H. Feshbach, Ann. Phys. (N.Y.) 5, 357 (1958)

${ }^{7}$ U. Fano, Phys. Rev. 124, 1866 (1961).

${ }^{8}$ (a) D. A. Micha, Adv. Quantum Chem. 8, 231 (1974); (b) D. A. Micha, in Modern Theoretical Chemistry, edited by W. H. Miller (Plenum, New York, 1976), Vol. IA, p. 81.

${ }^{9}$ L. S. Cederbaum and F. Tarantelli, J. Chem. Phys. 98, 9691 (1993).

${ }^{10}$ L. Allen and J. H. Eberly, Optical Resonance and Two-Level Atoms (Wiley, New York, 1975), Chap. 2.

${ }^{11}$ A. Bambini and P. R. Berman, Phys. Rev. A 23, 2496 (1981).

${ }^{12}$ G. Thomas, Phys. Rev. A 27, 2744 (1983).

${ }^{13}$ A. Salam and W. Meath, Chem. Phys. Lett. 277, 199 (1997).

${ }^{14}$ J. W. Gadzuk, in Vibrational Spectroscopy of Molecules on Surfaces, edited by J. T. Yates Jr. and T. E. Madey (Plenum, New York, 1987), p. 49.

${ }^{15}$ M. Head-Gordon and J. C. Tully, J. Chem. Phys. 96, 3939 (1992).

${ }^{16}$ T. A. Germer, J. C. Stephenson, E. J. Heilweil, and R. R. Cavanagh, J. Chem. Phys. 101, 1 (1994).

${ }^{17}$ J. W. Gadzuk, L. J. Richter, S. A. Buntin et al., Surf. Sci. 235, 317 (1990).

${ }^{18}$ J. A. Prybyla, H. W. K. Tom, and G. D. Aumiller, Phys. Rev. Lett. 68, 503 (1992).

${ }^{19}$ D. Beksic and D. A. Micha, J. Chem. Phys. 103, 3795 (1995); D. A. Micha and Z. Yi, J. Chem. Soc., Faraday Trans. 93, 969 (1997).

${ }^{20}$ D. A. Micha and Z-G. Yi, Chem. Phys. Lett. 298, 250 (1998); Z-G. Yi, D. A. Micha, and J. Sund, J. Chem. Phys. 110, 10562 (1999).

${ }^{21}$ A. Salam and D. A. Micha, Int. J. Quantum Chem. 75, 429 (1999).

${ }^{22}$ W. J. Brenig, Z. Phys. B: 23, 361 (1976).

${ }^{23}$ M. M. Sternheim and J. F. Walker, Phys. Rev. C 6, 114 (1972).

${ }^{24}$ D. A. McQuarrie, Statistical Mechanics (Harper and Row, New York, 1976), Chap. 21

${ }^{25}$ L. S. Rodberg and R. M. Thaler, Introduction to the Quantum Theory of Scattering (Academic, New York, 1967), Chap. 8.

${ }^{26}$ See, for example, Ref. 1, Chap. 3.

${ }^{27}$ Handbook of Mathematical Functions, edited by M. Abramowitz and I. A. Stegun (Dover, New York, 1965), Chap. 7. 